\title{
The Uniform Modulus of Continuity Of Iterated Brownian Motion ${ }^{1}$
}

\author{
By \\ Davar Khoshnevisan \\ Thomas M. Lewis \\ University of Utah $\mathcal{E}$ Furman University
}

Summary. Let $X$ be a Brownian motion defined on the line (with $\mathrm{X}(0)=0$ ) and let $Y$ be an independent Brownian motion defined on the nonnegative real numbers. For all $t \geq 0$, we define the iterated Brownian motion (IBM), $Z$, by setting $Z_{t} \triangleq X\left(Y_{t}\right)$. In this paper we determine the exact uniform modulus of continuity of the process $Z$.

Keywords and Phrases. Iterated Brownian motion, uniform modulus of continuity, the Ray-Knight theorem

Running Title. Iterated Brownian Motion.

A.M.S. Subject Classification (1991). 60F15; 60G17; $60 \mathrm{J65.}$

\footnotetext{
${ }^{1}$ Research supported by NSF grant DMS-9122242.
} 


\section{Introduction}

Let $X^{(1)}, X^{(2)}$ and $Y$ be independent standard Brownian motions starting from 0. Define a Brownian motion, $X$, on the line as follows:

$$
X(t) \triangleq \begin{cases}X^{(1)}(t) & \text { if } t \geq 0 \\ X^{(2)}(-t) & \text { if } t<0\end{cases}
$$

The iterated Brownian motion or iterated Wiener process, $Z$, is defined by setting $Z(t) \triangleq X(Y(t))$ for all $t \geq 0$. Iterated Brownian motions arise naturally in a variety of problems in probability and mathematical statistics. For example, Funaki $[\mathrm{F}]$ used a modification of $Z$ to give a probabilistic solution to the partial differential equation

$$
\frac{\partial^{4} u}{\partial x^{4}}=\frac{1}{8} \frac{\partial u}{\partial t} \quad \text { with } \quad u(0, x)=u_{0}(x)
$$

while Deheuvels and Mason [DH] introduced iterated Brownian motions in their study of the Bahadur-Kiefer process. Recent attention has centered on the path properties of $Z$. Burdzy [Bu1, Bu2] established the following local law of the iterated logarithm:

$$
\limsup _{t \rightarrow 0} \frac{Z(t)}{t^{1 / 4}(\ln \ln (1 / t))^{3 / 4}}=\frac{2^{5 / 4}}{3^{3 / 4}}, \quad \text { a.s. }
$$

Other notable results in this vein are: the functional form of (1.1) [HPS]; Chung's form of the law of the iterated logarithm [HPS] and [KL]; a characterization of the lower limits [S]; a characterization of the upper limits (via a connection with the stable subordinator of index 1/4) [Be]; various global Strassen-type theorems [CsCsFR1]; a study of the local time [CsCsFR2]; the Csörgö-Révész modulus of non-differentibility [HS]. (Some of the techniques of the present paper have played an important rôle in the analysis of [HS].)

Viewing (1.1) as the modulus of continuity of $Z$ at 0 , Burdzy (private communication) has asked whether one can find the uniform modulus of continuity for $Z$. The sole goal of this article is to answer this question. To this end define for all $\delta \in(0,1)$,

$$
\begin{aligned}
& \omega(\delta) \triangleq \sup _{0 \leq s, t \leq 1} \sup _{0 \leq|s-t| \leq \delta}|Z(s)-Z(t)| \\
& \psi(\delta) \triangleq \delta^{1 / 4}(\ln (1 / \delta))^{3 / 4} .
\end{aligned}
$$

Our theorem is the following: 
Theorem 1. With probability one,

$$
\lim _{\delta \rightarrow 0} \frac{\omega(\delta)}{\psi(\delta)}=1
$$

If we liberally estimate the modulus of $Z$ by composing the modulus of $X$ with that of $Y$, then, by the well-known result of Paul Lévy (see p. 114 of [KS]), we obtain:

$$
\limsup _{\delta \rightarrow 0} \omega(\delta) / \psi(\delta) \leq 2^{1 / 4}, \quad \text { a.s. }
$$

Since $2^{1 / 4}>1$, Theorem 1 shows that this simple argument yields the correct rate, but is not precise enough to anticipate the constant 1 . This, of course, is not unexpected, since the method outlined above is rather crude. What is surprising is that known general techniques, e.g., metric entropy and majorizing measures, are not refined enough to prove the upper bound in Theorem 1. Indeed, some estimates of Burdzy [Bu1] can be used to show that there exists $K>0$, such that for all $t, \varepsilon>0$ and $x>1$,

$$
\mathbb{P}\left(|Z(t+\varepsilon)-Z(t)| \geq \varepsilon^{1 / 4} x\right) \leq K \exp \left(-\chi x^{4 / 3}\right),
$$

where $\chi \triangleq 2^{5 / 3} \cdot 3^{-1}$. With this probability estimate and a direct imitation of Levy's uniform modulus argument for Brownian motion, one can at best obtain the following:

$$
\limsup _{\delta \rightarrow 0} \frac{\omega(\delta)}{\psi(\delta)} \leq \chi^{3 / 4}, \quad \text { a.s. }
$$

Since $\chi^{3 / 4} \approx 1.043>1$, even the upper bound (which is usually the easier half of such results) requires very new ideas. Our innovation is an analysis of IBM through the excursions of $Y$ from the fast points of $X$. To this end, one of the key results used is the Ray-Knight theorem (see the proof of Lemma 2.1).

\section{Preliminaries}

Throughout, we shall use generic constants $K, K_{1}$ and $K_{2}$; these may change from line to line (but not within the same line). Given positive functions $f$ and $g$, we will write $f(t) \sim g(t)$ as $t \rightarrow a$ to mean $\lim _{t \rightarrow a} f(t) / g(t)=1$.

Next we define two classes of stopping times for the Brownian motion, $Y$. For $a>0$ and $b \in \mathbb{R}$, let

$$
\begin{aligned}
\tau_{a} & =\tau(a) \triangleq \inf \left\{t>0:\left|Y_{t}\right|=a\right\} \\
\sigma_{b} & =\sigma(b) \triangleq \inf \left\{t>0: Y_{t}=b\right\} .
\end{aligned}
$$


By Brownian scaling, $a^{-2} \tau(a)$ and $b^{-2} \sigma(b)$ have the same distributions as $\tau(1)$ and $\sigma(1)$, respectively. We need the following well-known estimate for the small-ball probability of $\sigma(1)$ (see, e.g., p. 80 of $[\mathrm{KS}]$ ): as $t \rightarrow 0$,

$$
\mathbb{P}(\sigma(1) \leq t) \sim K t^{1 / 2} \exp \left(-\frac{1}{2 t}\right)
$$

By the symmetry of $Y$,

$$
\mathbb{P}(\sigma(1) \leq t) \leq \mathbb{P}(\tau(1) \leq t) \leq 2 \mathbb{P}(\sigma(1) \leq t)
$$

Therefore (2.1) implies the following:

$$
K_{1} t^{1 / 2} \exp \left(-\frac{1}{2 t}\right) \leq \mathbb{P}(\tau(1) \leq t) \leq K_{2} t^{1 / 2} \exp \left(-\frac{1}{2 t}\right)
$$

for all $t \leq 1$ and an appropriate choice of $K_{1}$ and $K_{2}$. Finally, by a theorem of Chung (see p. 221 of $[\mathrm{C}]$ ), as $s \rightarrow \infty$,

$$
\mathbb{P}(\tau(1) \geq s) \sim K \exp \left(-\frac{\pi^{2}}{8} s\right)
$$

Fixing an $R>1$, let $\mathbb{D}_{n} \triangleq\left\{k R^{-n}: k \in \mathbb{Z}\right\}$ and $r_{k, n} \triangleq k R^{-n}$, for all integers $k, n \geq 1$. Let $T_{0, n} \triangleq 0$ and iteratively define for $n, j \geq 1$,

$$
\begin{aligned}
& T_{j, n} \triangleq \inf \left\{s>T_{j-1, n}: Y(s) \in \mathbb{D}_{n} \backslash\left\{Y\left(T_{j-1, n}\right)\right\}\right\}, \\
& \Delta T_{j, n} \triangleq T_{j, n}-T_{j-1, n} .
\end{aligned}
$$

For integers $k, n \geq 1$, let us define the following sets of random indices:

$$
\begin{aligned}
& E_{k, n} \triangleq\left\{j \geq 1: Y\left(T_{j-1, n}\right)=r_{k-1, n}, Y\left(T_{j, n}\right)=r_{k, n} \text { and } T_{j, n} \leq 1\right\}, \\
& \widehat{E}_{k, n} \triangleq\left\{j \geq 1: Y\left(T_{j, n}\right)=r_{k, n} \text { and } T_{j, n} \leq 1\right\} .
\end{aligned}
$$

We point out that the cardinality of $E_{k, n}$ (denoted by $\# E_{k, n}$ ) is the number of times $Y$ upcrosses $\left[r_{k-1, n}, r_{k, n}\right]$ before time 1 .

Our next two lemmas are the most important of this section. In our first calculation, we demonstrate that with high probability, $Y$ upcrosses a significant number of the intervals $\left[r_{k-1, n}, r_{k, n}\right]$ a uniform number of times before time 1 . 
Lemma 2.1. Fix $0<\theta<\zeta<1$. Then there exists some $K=K(\theta, \zeta, R)>0$, such that for all integers $k, n \geq 1$,

$$
\mathbb{P}\left(\# E_{k, n} \leq R^{n(1-\zeta)} \text { for some } k \leq R^{n(1-2 \theta)}\right) \leq K n^{-2}
$$

Proof. Throughout, $L_{t}^{x}$ will denote the local time in $x$ of a standard Brownian motion. For simplicity, set $p_{n} \triangleq R^{-n \theta}$ and $M_{n} \triangleq R^{n(1-2 \theta)}$ and define the following events:

$$
\begin{aligned}
& \Omega_{0} \triangleq\left\{\# E_{k, n} \leq R^{n(1-\zeta)} \text { for some } k \leq M_{n}\right\} \\
& \Omega_{1} \triangleq\left\{4 L_{1}^{r_{k, n}} \geq R^{-n \zeta} \text { for all } 0 \leq k \leq M_{n}\right\} .
\end{aligned}
$$

It will suffice to demonstrate that $\mathbb{P}\left(\Omega_{1}^{c}\right)$ and $\mathbb{P}\left(\Omega_{0} \cap \Omega_{1}\right)$ are both bounded by $K n^{-2}$.

Clearly,

$$
\begin{aligned}
\mathbb{P}\left(\Omega_{1}^{c}\right) & \leq \mathbb{P}\left(\sigma\left(p_{n}\right) \geq 1\right)+\mathbb{P}\left(\Omega_{1}^{c}, \sigma\left(p_{n}\right) \leq 1\right) \\
& \triangleq I+I I
\end{aligned}
$$

notation being obvious. Now, $I$ is easily estimated: indeed, by the reflection principle,

$$
I=\mathbb{P}\left(\max _{0 \leq s \leq 1} Y_{s} \leq p_{n}\right)=\mathbb{P}\left(\left|Y_{1}\right| \leq p_{n}\right) \leq K p_{n}
$$

since $\left|Y_{1}\right|$ has a bounded density. Next we estimate $I I$. Note that $k \leq M_{n}$ implies that $r_{k, n} \leq p_{n}^{2}$. Since $\sigma\left(p_{n}\right) \leq 1$ and $t \mapsto L_{t}^{x}$ is nondecreasing, we obtain the bounds:

$$
\begin{aligned}
I I & \leq \mathbb{P}\left(\inf _{0 \leq r_{k, n} \leq p_{n}^{2}} L_{\sigma\left(p_{n}\right)}^{r_{k, n}} \leq \frac{1}{4} R^{-n \zeta}\right) \\
& \leq \mathbb{P}\left(\inf _{0 \leq x \leq p_{n}^{2}} L_{\sigma\left(p_{n}\right)}^{x} \leq \frac{1}{4} R^{-n \zeta}\right) \\
& =\mathbb{P}\left(\inf _{0 \leq x \leq p_{n}} L_{\sigma(1)}^{x} \leq \frac{1}{4} R^{-n(\zeta-\theta)}\right),
\end{aligned}
$$

where we have used scaling to obtain this last equality. Since $\sigma(1)$ is the first time $Y$ hits 1, it follows from the first Ray-Knight theorem (see Revuz and Yor [RY, Thm. 2.2, Ch. $\mathrm{XI}]$ ) that there exists a two-dimensional Brownian motion, $B$, such that $B(0)=0$ and $L_{\sigma(1)}^{1-x}=|B(x)|^{2}$ for all $0 \leq x \leq 1$. Therefore

$$
I I \leq \mathbb{P}\left(\inf _{1-p_{n} \leq x \leq 1}|B(x)| \leq 2 R^{-n(\zeta-\theta) / 2}\right) .
$$


This last probability can be estimated as follows: let $e_{n} \triangleq(0,1 / n) \in \mathbb{R}^{2}$. By the strong Markov property and a coupling argument, II is bounded above by

$$
\begin{aligned}
\mathbb{P} & \left(\left|B\left(1-p_{n}\right)\right| \leq n^{-1}\right)+\mathbb{P}\left(\inf _{0 \leq x \leq p_{n}}|B(x)| \leq 2 R^{-n(\zeta-\theta) / 2} \mid B(0)=e_{n}\right) \\
& \leq K n^{-2}+\mathbb{P}\left(\sup _{0 \leq x \leq p_{n}}|B(x)| \geq n^{-1}-2 R^{-n(\zeta-\theta) / 2} \mid B(0)=0\right) \\
& \left.\leq K n^{-2}+\mathbb{P}\left(\sup _{0 \leq x \leq p_{n}}|B(x)| \geq(2 n)^{-1} \mid B(0)=0\right) \quad \text { (for all large } n\right) \\
& =K n^{-2}+\mathbb{P}\left(\sup _{0 \leq x \leq 1}|B(x)| \geq(2 n)^{-1} \cdot R^{n \theta / 2}\right) \\
& \leq K n^{-2}+2 n R^{-n \theta / 2} \mathbb{E} \sup _{0 \leq x \leq 1}|B(x)| \\
& \leq K n^{-2},
\end{aligned}
$$

by Chebychev's inequality and the fact that $\mathbb{E} \sup _{0 \leq x \leq 1}|B(x)|<\infty$.

In light of the above development, we are left to estimate $\mathbb{P}\left(\Omega_{0} \cap \Omega_{1}\right)$. To do so, observe that

$$
\left\{\Omega_{0} \cap \Omega_{1}\right\} \subseteq \bigcup_{0 \leq k \leq M_{n}}\left\{2 R^{-n} \# E_{k, n} \leq(1 / 2) L_{1}^{r_{k, n}}\right\} .
$$

As a consequence of the estimates leading to (5.5) of $[\mathrm{K}], P\left(\Omega_{0} \cap \Omega_{1}\right)$ is bounded above by,

$$
K_{1}(n \ln R)^{-1 / 2} R^{-3 n}+\mathbb{P}\left(\sup _{x} L_{1}^{x} \leq 16 n^{2} R^{-2 n}(\ln R)^{2}\right) .
$$

To see this, take (in the notation of $[\mathrm{K}]$ ), $\theta=4, \zeta=1$ and $R=1$. You will see that $K_{1}$ above is the same as $C_{5}(4,1,1)$. Since $L_{1}^{0}$ has the same distribution as $|Y(1)|$, it has a bounded density. Hence, we certainly have, $\mathbb{P}\left(\Omega_{0} \cap \Omega_{1}\right) \leq K n^{-2}$, which is what we wished to show

In our next lemma, we show that the embedded random walk, $\left\{Y\left(T_{j, n}\right), j \geq 0\right\}$, does not hit an excessive number of points in $\mathbb{D}_{n}$ before time 1 . Furthermore, we show that no point in $\mathbb{D}_{n}$ gets hit too many times before time 1 .

Lemma 2.2. Let $\theta, \zeta \in(0,1)$. Then there exists some $K=K(R, \theta, \zeta)>0$ such that for all integers $n \geq 1$,

(1) $\mathbb{P}\left(\tau\left(R^{n \theta}\right) \leq 1\right) \leq K R^{-n \theta} \exp \left(-R^{2 n \theta} / 2\right)$

(2) $\mathbb{P}\left(\bigcup_{k \in \mathbb{Z}}\left\{\# \widehat{E}_{k, n}>R^{n(1+\zeta)}\right\}\right)$ is dominated by

$$
K_{1} R^{-n \theta} \exp \left(-R^{2 n \theta} / 2\right)+K_{2} \exp \left(-R^{n \zeta}\right)+K_{3} R^{n(1+\theta-\zeta / 2)} \exp \left(-R^{n \zeta} / 2\right) .
$$

Proof. We obtain (1) by (2.2) and scaling. Next we prove (2). 
Let $j=j(n) \triangleq\left[R^{n(2+\zeta)}\right]$. Observe that

$$
\mathbb{P}\left(\bigcup_{k \in \mathbb{Z}}\left\{\# \widehat{E}_{k, n}>R^{n(1+\zeta)}\right\}\right)
$$

is dominated by

$$
\mathbb{P}\left(\tau\left(R^{n \theta}\right) \leq 1\right)+\mathbb{P}\left(T_{j, n} \leq 1\right)+K R^{n(1+\theta)} \mathbb{P}\left(\xi(0, j(n))>R^{n(1+\zeta)}\right),
$$

where $\xi(0, \cdot)$ denotes the local time at 0 of a one-dimensional simple symmetric random walk. The first term has already been considered, and is given by (1).

To estimate the second term, we will need the Laplace transform of $\tau_{a}$ (see, for example, p. 67 of $[\mathrm{RY}])$ :

$$
\mathbb{E}\left(\exp \left(-\lambda \tau_{a}\right)\right)=(\cosh (a \sqrt{2 \lambda}))^{-1}
$$

Observe $T_{j, n}=\Delta T_{1, n}+\cdots+\Delta T_{j, n}$ can be written as the sum of $j(n)$ independent and identically distributed random variables, where $\Delta T_{1, n}$ has the same distribution as $\tau\left(R^{-n}\right)$. Thus, by Chebyshev's inequality and (2.4), we obtain the following:

$$
\mathbb{P}\left(T_{j, n} \leq 1\right) \leq e\left(\mathbb{E}\left(\exp \left(-\Delta T_{1, n}\right)\right)\right)^{j(n)}=e\left(\cosh \left(R^{-n} \sqrt{2}\right)\right)^{-j(n)}
$$

Since $\cosh (x) \geq 1+x^{2} / 2, \zeta<1$ and $\ln (1+x) \geq x-x^{2} / 2$ for $x \geq 0$, it follows that

$$
\mathbb{P}\left(T_{j, n} \leq 1\right) \leq K \exp \left(-R^{n \zeta}\right)
$$

Finally,

$$
\begin{aligned}
\mathbb{P}\left(\xi(0, j(n))>R^{n(1+\zeta)}\right) & =\mathbb{P}\left(j(n)^{-1 / 2} \xi(0, j(n))>R^{n \zeta / 2}\right) \\
& \leq K R^{-n \zeta / 2} \exp \left(-R^{n \zeta} / 2\right)
\end{aligned}
$$

for all $n$ sufficiently large, where we have used Bernstein's inequality or Chernoff's form of the large deviation estimate for the simple symmetric random walk and the fact that $\xi(0, n)$ has the same law as the absolute value of the simple symmetric random walk by time $n$ (see, e.g., p. 14 and p. 95 of $[\mathrm{R}]$ ).

Our next lemma provides a uniform upper bound on the spacings $\left\{\Delta T_{i, n}\right\}$ when $T_{i, n} \leq 1$.

Lemma 2.3. Let $\zeta>0$. Then there exist constants $K_{\ell}=K_{\ell}(R, \zeta)>0(\ell=1,2)$, such that for all integers $i, n \geq 1$,

$$
\mathbb{P}\left(\Delta T_{i, n} \geq n^{2} R^{-2 n} \text { for some } T_{i, n} \leq 1\right) \leq K_{1} \exp \left(-R^{n \zeta}\right)+K_{2} R^{n(2+\zeta)} \exp \left(-\pi^{2} n^{2} / 8\right)
$$


Proof. As in the proof of Lemma 2.2, let $j=j(n) \triangleq\left[R^{n(2+\zeta)}\right]$. Then the probability in question is dominated by

$$
\mathbb{P}\left(T_{j, n} \leq 1\right)+j(n) \mathbb{P}\left(\Delta T_{1, n} \geq n^{2} R^{-2 n}\right) .
$$

Using (2.5) to estimate the first term, it remains to bound the second term. By the strong Markov property and Brownian scaling,

$$
\begin{aligned}
\mathbb{P}\left(\Delta T_{1, n} \geq n^{2} R^{-2 n}\right) & =\mathbb{P}\left(\tau\left(R^{-n}\right) \geq n^{2} R^{-2 n}\right) \\
& =\mathbb{P}\left(\tau(1) \geq n^{2}\right)
\end{aligned}
$$

The result follows from (2.3) and (2.6).

\section{The proof of the lower bound}

Define for all $x \in(0,1)$,

$$
\begin{aligned}
& F(x) \triangleq \sqrt{x \ln (1 / x)}, \\
& G(x) \triangleq x^{2}(\ln (1 / x))^{-1} .
\end{aligned}
$$

Throughout, $\gamma$ and $\alpha$ will denote real numbers satisfying: $1 / 4<\gamma<1 / 2$ and

$$
0<\alpha<\sqrt{4-\gamma^{-1}}
$$

Given $\gamma$ and $\alpha$, let $\theta$ and $\zeta$ be chosen so that $0<\theta<\zeta<1$ and

$$
2-\frac{\alpha^{2}}{2}-\frac{1}{2 \gamma}>\zeta+2 \theta
$$

For convenience, let

$$
q \triangleq 2-2 \theta-\zeta-\frac{\alpha^{2}}{2}-\frac{1}{2 \gamma}>0
$$

For all $n \geq 1$ and $k \in \mathbb{Z}$, let us define the following random variables and events:

$$
\begin{aligned}
\Delta X_{k, n} & =X\left(r_{k, n}\right)-X\left(r_{k-1, n}\right) \\
A_{k, n} & \triangleq\left\{\left|\Delta X_{k, n}\right| \geq \alpha F\left(R^{-n}\right)\right\}, \\
B_{k, n} & \triangleq\left\{\Delta T_{j, n} \leq \gamma G\left(R^{-n}\right) \text { for some } j \in E_{k, n}\right\}, \\
C_{k, n} & \triangleq A_{k, n} \cap B_{k, n} .
\end{aligned}
$$


Our goal is to show that at least one of the $C_{k, n}$ occurs for some index $k$ with $T_{k, n} \leq 1$, almost surely and for all $n$ sufficiently large. To this end, our first goal is to establish the following: for all $\alpha, \gamma, \theta$ and $\zeta$ satisfying (3.1), we have

$$
\sum_{n=1}^{\infty} \mathbb{P}\left(\bigcap_{\left\{k: T_{k, n} \leq 1\right\}}\left(C_{k, n}\right)^{c}\right)<\infty .
$$

For each $k, n \geq 1$, let us define the set of random indices:

$$
\mathcal{E}_{k, n} \triangleq\left\{j \geq 1: Y\left(T_{j-1, n}\right)=r_{k-1, n}, Y\left(T_{j, n}\right)=r_{k, n}\right\} .
$$

Thus $\mathcal{E}_{k, n}$ is the set of indices $j$ that correspond to upcrossings of the interval $\left[r_{k-1, n}, r_{k, n}\right]$. This is distinguished from $E_{k, n}$ in that we have no restrictions on time. For $0 \leq k \leq$ $\left[R^{n(1-2 \theta)}\right]$, define the measurable event,

$$
\mathcal{B}_{k, n} \triangleq\left\{\Delta T_{j, n}>\gamma G\left(R^{-n}\right) \text { for the first }\left[R^{n(1-\zeta)}\right] \text { elements of } \mathcal{E}_{k, n}\right\} .
$$

In words, this is the event that the time for each of the the first $\left[R^{n(1-\zeta)}\right]$ upcrossings of the interval $\left[r_{k-1, n}, r_{k, n}\right]$ by $Y$ exceeds $\gamma G\left(R^{-n}\right)$. We observe that

$$
B_{k, n}^{c} \cap\left\{\# E_{k, n}>R^{n(1-\zeta)} \text { for all } 0 \leq k \leq R^{n(1-2 \theta)}\right\} \subset \mathcal{B}_{k, n}
$$

By the strong Markov property, the events $\left\{A_{k, n}^{c} \cup \mathcal{B}_{k, n}, 0 \leq k \leq R^{n(1-2 \theta)}\right\}$ are independent and

$$
\mathbb{P}\left(A_{k, n}^{c} \cup \mathcal{B}_{k, n}\right)=\mathbb{P}\left(A_{1, n}^{c} \cup \mathcal{B}_{1, n}\right) .
$$

These considerations lead us to the estimate:

$$
\begin{aligned}
\mathbb{P}\left(\bigcap_{\left\{k: T_{k, n} \leq 1\right\}}\left(C_{k, n}\right)^{c}\right) \leq & \mathbb{P}\left(\# E_{k, n} \leq R^{n(1-\zeta)} \text { for some } k \leq R^{n(1-2 \theta)}\right) \\
& +\mathbb{P}\left(\bigcap_{k \leq R^{n(1-2 \theta)}}\left(A_{k, n}^{c} \cup \mathcal{B}_{k, n}\right)\right) \\
& \leq K \cdot n^{-2}+\mathbb{P}\left(\bigcap_{k \leq R^{n(1-2 \theta)}}\left(A_{k, n}^{c} \cup \mathcal{B}_{k, n}\right)\right)
\end{aligned}
$$

by Lemma 2.1. Since the events $A_{k, n}$ and $\mathcal{B}_{k, n}$ are independent,

$$
\begin{aligned}
\mathbb{P}\left(\bigcap_{k \leq R^{n(1-2 \theta)}}\left(A_{k, n}^{c} \cup \mathcal{B}_{k, n}\right)\right) & \leq\left(1-\mathbb{P}\left(A_{1, n}\right) \mathbb{P}\left(\mathcal{B}_{1, n}^{c}\right)\right)^{\left[R^{n(1-2 \theta)}\right]} \\
& \leq \exp \left(-\left[R^{n(1-2 \theta)}\right] \mathbb{P}\left(A_{1, n}\right) \mathbb{P}\left(\mathcal{B}_{1, n}^{c}\right)\right)
\end{aligned}
$$


By scaling and a standard calculation, we readily obtain:

$$
\mathbb{P}\left(A_{1, n}\right)=P\left(|X(1)| \geq \alpha R^{n / 2} F\left(R^{-n}\right)\right) \geq K n^{-1 / 2} R^{-n \alpha^{2} / 2} .
$$

By the strong Markov property and (2.2),

$$
\begin{aligned}
\mathbb{P}\left(\mathcal{B}_{1, n}^{c}\right) & =1-\left(1-\mathbb{P}\left(\Delta T_{1, n} \leq \gamma G\left(R^{-n}\right)\right)\right)^{\left[R^{n(1-\zeta)}\right]} \\
& \geq 1-\left(1-K n^{-1 / 2} R^{-n /(2 \gamma)}\right)^{\left[R^{n(1-\zeta)}\right]} \\
& \geq K n^{-1 / 2} R^{n(1-\zeta-1 /(2 \gamma))}
\end{aligned}
$$

since, by definition, $(2 \gamma)^{-1}>1-\zeta$.

Recalling the definition of $q>0$ from (3.1), we see from (3.3), (3.4) and (3.5) that

$$
\mathbb{P}\left(\bigcap_{k \leq R^{n(1-2 \theta)}}\left(A_{k, n}^{c} \cup \mathcal{B}_{k, n}\right)\right) \leq \exp \left(-K n^{-1} R^{n q}\right) .
$$

Hence, from (3.2) we obtain the estimate:

$$
\mathbb{P}\left(\bigcap_{\left\{k: T_{k, n} \leq 1\right\}}\left(C_{k, n}\right)^{c}\right) \leq K_{1} n^{-2}+\exp \left(-K_{2} n^{-1} R^{n q}\right),
$$

which certainly sums.

By the Borel-Cantelli lemma there exists an an $N_{0}$ such that for all $n \geq N_{0}$ there exists $k=k(n)$ such that

$$
\left|\Delta X_{k, n}\right| \geq \alpha F\left(R^{-n}\right) \quad \text { and } \quad \Delta T_{k, n} \leq \gamma G\left(R^{-n}\right)
$$

on a set of full measure. We point out the elementary fact that $G(\cdot)$ is invertible on $(0,1)$ and that $F\left(G^{-1}(x)\right) \sim 2^{-3 / 4} \psi(x)$ as $x \rightarrow 0^{+}$, where $G^{-1}$ is the inverse function to $G$. Moreover the following asymptotic relationships are easily verified:

$$
\begin{array}{rlrl}
F\left(R^{-n}\right) & \sim R^{-1 / 2} F\left(R^{-n+1}\right) \quad \text { as } n \rightarrow \infty ; & \\
F\left(R^{-n+1}\right) & =F\left(G^{-1}\left(G\left(R^{-n+1}\right)\right)\right) \sim 2^{-3 / 4} \cdot \psi\left(G\left(R^{-n+1}\right)\right) & & \text { as } n \rightarrow \infty ; \\
\psi(x) & \sim \gamma^{-1 / 4} \psi(\gamma x) \quad \text { as } x \rightarrow 0^{+} . &
\end{array}
$$

Given any $\epsilon>0$, from these considerations and (3.6) it follows that there almost surely exists an $N_{1} \geq N_{0}$ such that for all $n \geq N_{1}$ there exists a $k=k(n)$ for which

$$
\Delta X_{k, n} \geq(1-\varepsilon) 2^{-3 / 4} \alpha \gamma^{-1 / 4} R^{-1 / 2} \psi\left(\gamma G\left(R^{-n+1}\right)\right) \quad \text { and } \quad \Delta T_{k, n} \leq \gamma G\left(R^{-n}\right) .
$$


Since $\Delta X_{k, n}=Z\left(T_{k, n}\right)-Z\left(T_{k-1, n}\right)$, then for any $n \geq N_{1}$ we have

$$
\omega\left(\gamma G\left(R^{-n}\right)\right) \geq(1-\varepsilon) 2^{-3 / 4} \alpha \gamma^{-1 / 4} R^{-1 / 2} \psi\left(\gamma G\left(R^{-n+1}\right)\right) .
$$

For any $\delta>0$ small enough, there exists $n \geq N_{1}$ such that

$$
\gamma G\left(R^{-(n+1)}\right) \leq \delta \leq \gamma G\left(R^{-n}\right) .
$$

By monotonicity, for such a $\delta$,

$$
\begin{aligned}
\omega(\delta) & \geq \omega\left(\gamma G\left(R^{-(n+1)}\right)\right) \\
& \geq(1-\varepsilon) 2^{-3 / 4} \alpha \gamma^{-1 / 4} R^{-1 / 2} \psi\left(\gamma G\left(R^{-n}\right)\right) \\
& \geq(1-\varepsilon) 2^{-3 / 4} \alpha \gamma^{-1 / 4} R^{-1 / 2} \psi(\delta) .
\end{aligned}
$$

Therefore,

$$
\liminf _{\delta \rightarrow 0} \frac{\omega(\delta)}{\psi(\delta)} \geq(1-\varepsilon) 2^{-3 / 4} \alpha \gamma^{-1 / 4} R^{-1 / 2}, \quad \text { a.s.. }
$$

Let $\varepsilon \downarrow 0$ and $R \downarrow 1$ to obtain the following:

$$
\liminf _{\delta \rightarrow 0} \frac{\omega(\delta)}{\psi(\delta)} \geq 2^{-3 / 4} \alpha \gamma^{-1 / 4}, \quad \text { a.s.. }
$$

Let $\theta, \zeta \downarrow 0, \gamma \rightarrow 1 / 2$ and $\alpha \rightarrow \sqrt{2}$ to obtain the promised lower bound.

\section{The proof of the upper bound}

Throughout let $R>1, \theta, \zeta, q \in(0,1)$. We will continue to use the notation developed in Section 2 , significantly the family of stopping times $\left\{T_{i, n}\right\}$.

First we will need some technical definitions. Let $I \triangleq[a, b]$ denote a generic closed interval with endpoints in $\mathbb{D}_{n}$ and let $|I| \triangleq b-a$ denote its length. For $\alpha \geq 0$, we say that $I$ is $\alpha$-fast (written $I \in \mathcal{F}^{\alpha}$ ) provided that

$$
|X(b)-X(a)| \geq \alpha F(|I|) .
$$

By a standard Brownian motion calculation, there exists some $K=K(R, \alpha)>0$ such that for any interval, $I \subseteq \mathbb{R}_{+}^{1}$,

$$
\mathbb{P}\left(I \in \mathcal{F}^{\alpha}\right) \sim K\left(\log \left(|I|^{-1}\right)\right)^{-1 / 2}|I|^{\alpha^{2} / 2} \quad \text { as }|I| \rightarrow 0 .
$$

We will call a pair of stopping times $\left(T_{i, n}, T_{l, n}\right)$ (with $j<l$ ) a crossing of $I$ provided that either 
(i) $Y\left(T_{i, n}\right)=a, Y\left(T_{l, n}\right)=b$ and $Y\left(T_{k, n}\right)<b$ for all indices $k$ satisfying $j<k<l$; or

(ii) $Y\left(T_{i, n}\right)=b, Y\left(T_{l, n}\right)=a$ and $Y\left(T_{k, n}\right)>a$ for all indices $k$ satisfying $j<k<l$.

Corresponding to a crossing $\left(T_{i, n}, T_{l, n}\right)$ of $I$, we define the crossing time of $I$ as simply $T_{l, n}-T_{i, n}$.

We will need the following collection of intervals: for $n \geq 1$ and $1 \leq j \leq n$ let

$$
\mathcal{I}_{j, n} \triangleq\left\{\left[r_{k, n}, r_{k+j, n}\right]:|k| \leq R^{n(1+\theta)}+1\right\} .
$$

Finally, for $0 \leq \alpha<2$ let

$$
\gamma=\gamma(\alpha) \triangleq \frac{1}{4-\alpha^{2}+2 q+2 \theta+2 \zeta} .
$$

For future reference, we note that

$$
q \triangleq \frac{\alpha^{2}}{2}+\frac{1}{2 \gamma}-2-\zeta-\theta>0
$$

Let $\mathcal{A}_{n}$ be the event that before time 1 at least one interval of the form $\left[r_{k, n}, r_{k+j, n}\right]$ (with $1 \leq j \leq n$ ) which is in $\mathcal{F}^{\alpha}$ is crossed in time less than $\gamma G\left(j R^{-n}\right)$ by $Y$. Likewise, for $n \geq 1$ and $1 \leq j \leq n$, let $\mathcal{A}_{j, n}$ denote the event that at least one of the intervals from $\mathcal{I}_{j, n}$ which is in $\mathcal{F}^{\alpha}$ is crossed in time less than $\gamma G\left(j R^{-n}\right)$ by one of the first $R^{n(1+\zeta)}$ crossings of that interval.

Let $l<k$ be integers and suppose that $\# \widehat{E}_{l, n} \leq R^{n(1+\zeta)}$ and $\# \widehat{E}_{k, n} \leq R^{n(1+\zeta)}$. Then the number of crossings of the interval $\left[r_{l, n}, r_{k, n}\right]$ by $Y$ up to time 1 is bounded above by $R^{n(1+\zeta)}$. Similarily, if $\tau\left(R^{n \theta}\right)>1$, then $|Y(t)| \leq r_{k, n}$ for all $|k| \leq R^{n(1+\theta)}+1$ and $0 \leq t \leq 1$. Consequently,

$$
\mathcal{A}_{n} \cap\left\{\tau\left(R^{n \theta}\right)>1\right\} \cap\left(\bigcup_{k \in \mathbb{Z}}\left\{\# \widehat{E}_{k, n}>R^{n(1+\zeta)}\right\}\right)^{c} \subset \bigcup_{j=1}^{n} \mathcal{A}_{j, n} .
$$

Thus, by subadditivity,

$$
\mathbb{P}\left(\mathcal{A}_{n}\right) \leq a_{n}+\sum_{j=1}^{n} \mathbb{P}\left(\mathcal{A}_{j, n}\right),
$$

where

$$
a_{n} \triangleq \mathbb{P}\left(\tau\left(R^{n \theta}\right) \leq 1\right)+\mathbb{P}\left(\bigcup_{k \in \mathbb{Z}}\left\{\# \widehat{E}_{k, n}>R^{n(1+\zeta)}\right\}\right) .
$$

By Lemma 2.2, $a_{n}$ is summable; we are left to estimate $\mathbb{P}\left(\mathcal{A}_{j, n}\right)$. 
Since $X$ and $Y$ are independent, by conditioning on the number of intervals in $\mathcal{I}_{j, n} \cap$ $\mathcal{F}^{\alpha}$, then applying subadditivity and integrating, $\mathbb{P}\left(\mathcal{A}_{j, n}\right)$ is bounded above by the product of the following three terms:

(i) the expected number of intervals in $\mathcal{I}_{j, n} \cap \mathcal{F}^{\alpha}$;

(ii) $R^{n(1+\zeta)}$ (i.e., the number of observed crossings per interval);

(iii) the probability that the time to cross an interval with length $j / R^{n}$ is faster than $\gamma G\left(j / R^{n}\right)$.

By (4.1), we see that the probability that a given interval in $\mathcal{I}_{j, n}$ is in $\mathcal{F}^{\alpha}$ is bounded above by $K n^{1 / 2} R^{-n \alpha^{2} / 2} j^{\alpha^{2} / 2}$. Since the number of intervals in $\mathcal{I}_{j, n}$ is asymptotically $2 R^{n(1+\theta)}$, it follows that the expected number of intervals in $\mathcal{I}_{j, n} \cap \mathcal{F}^{\alpha}$ is bounded above by

$$
K n^{1 / 2} R^{n\left(1+\theta-\alpha^{2} / 2\right)} j^{\alpha^{2} / 2} .
$$

This estimates the term described in (i), and it remains to estimate the probability in (iii); however, by (2.1), this probability is bounded above by

$$
K n^{1 / 2} R^{-n /(2 \gamma)} j^{1 /(2 \gamma)}
$$

It follows that

$$
\mathbb{P}\left(\mathcal{A}_{j, n}\right) \leq K n R^{n\left(2+\theta+\zeta-\alpha^{2} / 2-1 /(2 \gamma)\right)} j^{\alpha^{2} / 2+1 /(2 \gamma)} .
$$

By (4.4),

$$
2+\theta+\zeta-\frac{\alpha^{2}}{2}-\frac{1}{2 \gamma}=-q .
$$

Moreover, since $q, \gamma, \theta \in(0,1),(4.4)$ yields:

$$
\frac{\alpha^{2}}{2}+\frac{1}{2 \gamma}=2+q+\gamma+\theta \leq 5
$$

Consequently,

$$
\mathbb{P}\left(\mathcal{A}_{j, n}\right) \leq K n R^{-n q} j^{5}
$$

and

$$
\sum_{j=1}^{n} \mathbb{P}\left(\mathcal{A}_{j, n}\right) \leq K n^{7} R^{-n q},
$$

which certainly sums in $n$, since $q>0$. We have shown that $\sum_{n} P\left(\mathcal{A}_{n}\right)<\infty$. By the Borel-Cantelli lemma, it follows that

$$
\mathbb{P}\left(\mathcal{A}_{n}, \text { i.o. }\right)=0
$$


Hence on a set of full measure and for $n$ sufficiently large, we can make the following observation: let $0 \leq i<j$ be integers and suppose that $T_{j, n} \leq 1$. Let $a \triangleq Y\left(T_{i, n}\right) \wedge Y\left(T_{j, n}\right)$ and $b \triangleq Y\left(T_{i, n}\right) \vee Y\left(T_{j, n}\right)$. Whenever

$$
b-a \leq \frac{n}{R^{n}} \quad \text { and } \quad|X(b)-X(a)| \geq \alpha F(b-a),
$$

it too must be the case that

$$
T_{j, n}-T_{i, n} \geq \gamma(\alpha) G(b-a) .
$$

Indeed, by (4.6), the interval $[a, b]$ determined by $T_{i, n}$ and $T_{j, n}$ is smaller (in length) than $n / R^{n}$ and is in $\mathcal{F}^{\alpha}$; thus by (4.5), $T_{j, n}-T_{i, n}$ must exceed some time to cross this interval, which, in turn, must exceed $\gamma(\alpha) G(b-a)$.

Fix an integer $m \geq 1$ and let $\alpha_{k}=k \sqrt{2} / m$ for $0 \leq k \leq m+1$. This determines $(m+1)$ intervals, $\left[\alpha_{0}, \alpha_{1}\right] \ldots\left[\alpha_{m}, \alpha_{m+1}\right]$. Corresponding to each of the latter intervals, by (4.3), we get $(m+1)$ values of $\gamma: \gamma\left(\alpha_{0}\right), \ldots, \gamma\left(\alpha_{m}\right)$. Let

$$
C_{m} \triangleq 2^{-3 / 4} \max \left\{\frac{\alpha_{1}}{\gamma^{1 / 4}\left(\alpha_{0}\right)}, \ldots, \frac{\alpha_{m+1}}{\gamma^{1 / 4}\left(\alpha_{m}\right)}\right\} .
$$

Let $T_{i, n}, T_{j, n}, a$ and $b$ be as in the previous paragraph. By the usual modulus of continuity of $X$,

$$
\alpha_{k} F(b-a) \leq|X(b)-X(a)|<\alpha_{k+1} F(b-a),
$$

for some $0 \leq k \leq m$. Thus $[a, b]$ is in $\mathcal{F}^{\alpha_{k}}$, for some $\alpha_{k}$. Consequently, provided that $b-a \leq n / R^{n}$, it follows that

$$
T_{j, n}-T_{i, n} \geq \gamma\left(\alpha_{k}\right) G(b-a) \quad \text { and } \quad|X(b)-X(a)| \leq \alpha_{k+1} F(b-a) .
$$

We recall that $F\left(G^{-1}(x)\right) \sim 2^{-3 / 4} \psi(x)$ and $\psi(\gamma x) \sim \gamma^{-1 / 4} \psi(x)$ as $x \rightarrow 0^{+}$. Let $\epsilon>0$ be given. Then for $x \geq 0$ sufficiently small, $F\left(G^{-1}(\gamma x)\right) \leq(1+\epsilon) 2^{-3 / 4} \gamma^{-1 / 4} \psi(x)$. Thus, assuming that $b-a \leq n / R^{n}$, we have shown:

$$
\left|Z\left(T_{j, n}\right)-Z\left(T_{i, n}\right)\right| \leq(1+\epsilon) C_{m} \psi\left(T_{j, n}-T_{i, n}\right) .
$$

Finally, let $\delta>0$ satisfy

$$
\frac{n+1}{R^{n+1}} \leq 2 \sqrt{\delta \log \left(\delta^{-1}\right)} \leq \frac{n}{R^{n}} .
$$

Let $T_{i, n}<T_{j, n} \leq 1$ with $T_{j, n}-T_{i, n} \leq \delta$. Then, by the uniform modulus of continuity of $Y$, it follows that

$$
\left|Y\left(T_{j, n}\right)-Y\left(T_{i, n}\right)\right| \leq 2 \sqrt{\delta \log \left(\delta^{-1}\right)} \leq \frac{n}{R^{n}}
$$


which shows that the interval $[a, b]=\left[Y\left(T_{i, n}\right) \wedge Y\left(T_{j, n}\right), Y\left(T_{i, n}\right) \vee Y\left(T_{j, n}\right)\right]$ is not too wide. Thus we have shown that whenever $T_{i, n}<T_{j, n} \leq 1$ with $T_{j, n}-T_{i, n} \leq \delta$, then

$$
\left|Z\left(T_{j, n}\right)-Z\left(T_{i, n}\right)\right| \leq(1+\epsilon) C_{m} \psi\left(T_{j, n}-T_{i, n}\right)
$$

The above is the desired upper bound for the modulus of continuity of $Z$ along the random subsequence given by $\left\{T_{i, n} ; i\right\}$. We finish the proof of the upper bound (and hence that of Theorem 1) by chaining deterministic times to the subsequence $\left\{T_{i, n} ; i\right\}$ and showing that the "slack" in this chaining approximation is negligible.

Given $0 \leq s<t \leq 1$ with $t-s \leq \delta$, either

(i) $t-s \leq n^{2} / R^{2 n}$, in which case we can use (1.2) (i.e., the inexact modulus of continuity of $Z$ ) and conclude that for some $K>0$,

$$
|Z(t)-Z(s)| \leq K \psi\left(n^{2} / R^{2 n}\right) ; \text { or }
$$

(ii) $n^{2} / R^{n}<t-s \leq \delta$. In this case, using Lemma 2.3, for all $n$ sufficiently large, $\Delta T_{i, n} \leq$ $n^{2} / R^{2 n}$ for all $T_{i, n} \leq 1$. It is a simple task to see that there exists $i$ such that $1-$ $n^{2} R^{-2 n} \leq T_{i, n} \leq 1$. (Use time-reversal and the proof of Lemma 2.3, for instance.) Thus, we can find $i$ and $j$, such that $s \leq T_{i, n} \leq T_{j, n} \leq t, T_{i, n}-s \leq n^{2} / R^{2 n}$ and $t-T_{j, n} \leq n^{2} / R^{2 n}$.

Thus, by the triangle inequality, (1.2), (4.7) and (4.8), it follows that

$$
\begin{aligned}
|Z(t)-Z(s)| \leq\left|Z(t)-Z\left(T_{i, n}\right)\right|+\left|Z(s)-Z\left(T_{j, n}\right)\right|+ \\
\quad+\left|Z\left(T_{i, n}\right)-Z\left(T_{j, n}\right)\right| \\
\leq K \psi\left(n^{2} / R^{2 n}\right)+(1+\epsilon) C_{m} \psi\left(T_{j, n}-T_{i, n}\right) \\
\leq K \psi\left(n^{2} / R^{2 n}\right)+(1+\epsilon) C_{m} \psi(t-s) \\
\leq K \psi\left(n^{2} / R^{2 n}\right)+(1+\epsilon) C_{m} \psi(\delta) .
\end{aligned}
$$

Therefore,

$$
\omega(\delta) \leq K \psi\left(n^{2} / R^{2 n}\right)+(1+\epsilon) C_{m} \psi(\delta) .
$$

However, by (4.7), $\delta \sim K n^{9} / R^{2 n}$ as $n \rightarrow \infty$. This shows that $\psi\left(n^{2} / R^{2 n}\right)=o(\psi(\delta))$ and from this observation, it follows that

$$
\limsup _{\delta \rightarrow 0^{+}} \frac{\omega(\delta)}{\psi(\delta)} \leq(1+\epsilon) C_{m} \quad \text { a.s. }
$$


We are done since since $\epsilon>0$ is arbitrary and, by (4.3) and the definitions of $C_{m}$ and $\alpha_{m}$,

$$
\lim _{m \rightarrow \infty} \lim _{\zeta \rightarrow 0} \lim _{\theta \rightarrow 0} \lim _{q \rightarrow 0} C_{m}=\sup _{\alpha \in[0, \sqrt{2}]} \frac{\alpha\left(4-\alpha^{2}\right)^{1 / 4}}{2^{3 / 4}}=1 .
$$

This finishes the proof of the upper bound and hence that of Theorem 1.

Acknowledgements. Part of this work was carried out while we were visiting the Center for the Mathematical Sciences at the University of Wisconsin-Madison. We wish to extend our thanks to Tom Kurtz and Jim Kuelbs for their hospitality, and to the National Science Foundation for their financial support. Many thanks are due to Chris Burdzy for suggesting this problem, as well as his continued interest. Our whole-hearted thanks go to Zhan Shi for carefully reading and correcting the first draft of this paper.

\section{References}

[Be] J. Bertoin (1995). Iterated Brownian motion and stable $1 / 4$ subordinator. To appear in Stat. Prob. Lett.

[Bu1] K. Burdzy (1993). Some path properties of iterated Brownian motion. Sem. Stoch. Processes 1992, K.L. Chung, E. Cinlar and M.J. Sharpe, eds., Birkhauser 1993, pp. 67-87.

[Bu2] K. Burdzy (1993). Variation of iterated Brownian motion. Measure-valued Processes, Stochastic Partial Differential Equations and Interacting Particle Systems, (Ed. D.A. Dawson) CRM Proceedings and Lecture Notes, 5, 35-53.

[C] K.L. Chung (1948). On the maximum of partial sums of sequences of independent random variables. Trans. Amer. Math. Soc., 64, pp. 205-233.

[CsCsFR1] E. Csáki, M. Csörgo, A. Földes and P. Révész (1995). Global Strassen-type theorems for iterated Brownian motion. To appear in Stoch. Process. Appl.

[CsCsFR2] E. Csáki, M. Csörgo, A. Földes and P. Révész (1994). The local time of iterated Brownian motion. Technical Report Series of the Laboratory for Research in Statatistic and Probability, 250.

[F] T. Funaki (1979). Probabilistic construction of the solution of some higher order parabolic differential equations, Proc. Japan Acad. 55, 176-179

[HS] Y. Hu and Z. Shi (1994). The Csörgo-Révész modulus of non-differentiability of iterated Brownian motion. To appear in Stoch. Process. Appl. 
[HPS] Y. Hu, D. Pierre-Loti-Viaud and Z. Shi (1994) Laws of the iterated logarithm for iterated Wiener processes. To appear in J. Theor. Probab.

[K] D. Khoshnevisan (1993). Exact rates of convergence to Brownian local time. Ann. Prob. 22 (3), 1295-1330.

[KL] D. Khoshnevisan and T. Lewis (1993). Chung's law of the iterated logarithm for iterated Brownian motion. To appear in Ann. Inst. Henri Poincaré: Prob. et Stat.

[KS] I. Karatzas and S. Shreve (1988). Brownian motion and Stochastic Calculus. Springer Verlag, New York.

[R] P. Révész (1990). Random Walk in Random and Non-Random Environments. World Scientific, Singapore.

[RY] D. Revuz and M. Yor (1991). Continuous Martingales and Brownian Motion. Springer Verlag, Berlin-Heidelberg.

[S] Z. Shi (1994) Lower limits of iterated Wiener process. To appear in Stat. Prob. Lett.

D. Khoshnevisan

Department of Mathematics

University of Utah

Salt Lake City, UT 84112
T.M. Lewis

Department of Mathematics

Furman University

Greenville, SC 29613 